\title{
Differential Effects of the Rod-and-Frame Illusion on the Timing of Forearm Rotations
}

\author{
Janneke Lommertzen, Alexander M.J. van Zuijlen, \\ Ruud G.J. Meulenbroek, and Rob van Lier
}

\begin{abstract}
The current study focused on the time course of the effects of the rod-and-frame illusion (RFI) on the kinematics of targeted forearm rotations. Participants were asked to reproduce perceived rod orientations by propelling a hand-held cylinder forward while rotating it to the target orientation. Rod and frame orientations were systematically varied, and cylinder rotations were normalized to time. Average realized cylinder orientations confirmed that when the frame orientation deviated from the vertical, a reproduction error occurred in the direction opposite to the direction of the frame tilt. In contrast, the perceived orientation of the stimulus rod was exaggerated relative to the vertical (i.e., reproduction errors were in the direction of the rod tilt). Furthermore, linear regression analyses for every normalized time sample showed that the rod and frame effects start simultaneously, but they reach their maximum effect at different points in time. We discuss the implications of our findings for current views on the effects of visual illusions on motor control.
\end{abstract}

Keywords: kinematics, prehension, visuomotor coordination, orientation

Much research examining interactions between perception and action has recently focused on behavioral effects of visual illusions on motor performance. In most of these studies participants were asked to make pointing (van Donkelaar, 1999; Fischer, 2001) or grasping responses (Aglioti, Desouza, \& Goodale, 1995; Franz, Gegenfurtner, Bülthoff, \& Fahle, 2000; Jackson \& Shaw, 2000; Franz, Fahle, Bülthoff, \& Gegenfurtner, 2001) in the context of size-contrast illusions such as the Ebbinghaus illusion (Aglioti et al., 1995; Franz et al., 2001), the Brentano- or Müller-Lyer illusion (De Grave, Brenner, \& Smeets, 2004; Heath, Rival, \& Binsted, 2004), or the Ponzo illusion (van Donkelaar, 1999; Jackson \& Shaw, 2000). Usually, pointing or aperture errors were used as critical performance indices in these studies.

In the current study we investigated the effects of the two constituent parts of an orientation illusion. Rather than focusing on perception-action interactions as such, we zoomed in on the time course of the illusion's effects on forearm rota-

The authors are with Radboud University Nijmegen, Donders Institute for Brain, Cognition and Behaviour, 6500 HE Nijmegen, The Netherlands. 
tions. For this purpose we exploited the rod-and-frame illusion (RFI), which is elicited by a visual stimulus consisting of a straight line segment surrounded by a rectangular frame. The illusion is caused when the frame is rotated from vertical, which biases the perceived orientation of the rod (Beh, Wenderoth, \& Purcell, 1971). The effects of the RFI depend mainly on the amount of frame tilt (Antonucci, Fanzon, Spinelli, \& Zoccolotti, 1995; Beh \& Wenderoth, 1972). The marked effect the rotated frames have on the perceived target orientations of the rods can already be experienced by a quick glance at our stimuli in Figure 1. The RFI is, in a way, the rotational analog of the so-called induced Roelofs effect, in which a laterally shifted frame biases delayed pointing responses toward a target position within that surrounding frame (Bridgeman, Peery, \& Anand, 1997).

In an earlier attempt to capture the time course of orientation illusions, the RFI was used in combination with a simultaneous tilt illusion (STI), which consisted of a circular target grating surrounded by a larger circular grating (Dyde \& Milner, 2002). The two illusions were tested in two task types categorized as being either "perceptual" or "goal directed" in nature. The first task type involved either matching the orientation of the target stimulus with a second rod that was placed next to the stimulus within a separate frame or rotating a separate grating that was located below the stimulus. The second task type was either to grasp the target rod of the RFI or, in the experiments with the STI, to "mail" a card against the "slots" of the grating they saw before. Surprisingly, whereas the STI effects were found in both task types, RFI effects were only found in the orientation-

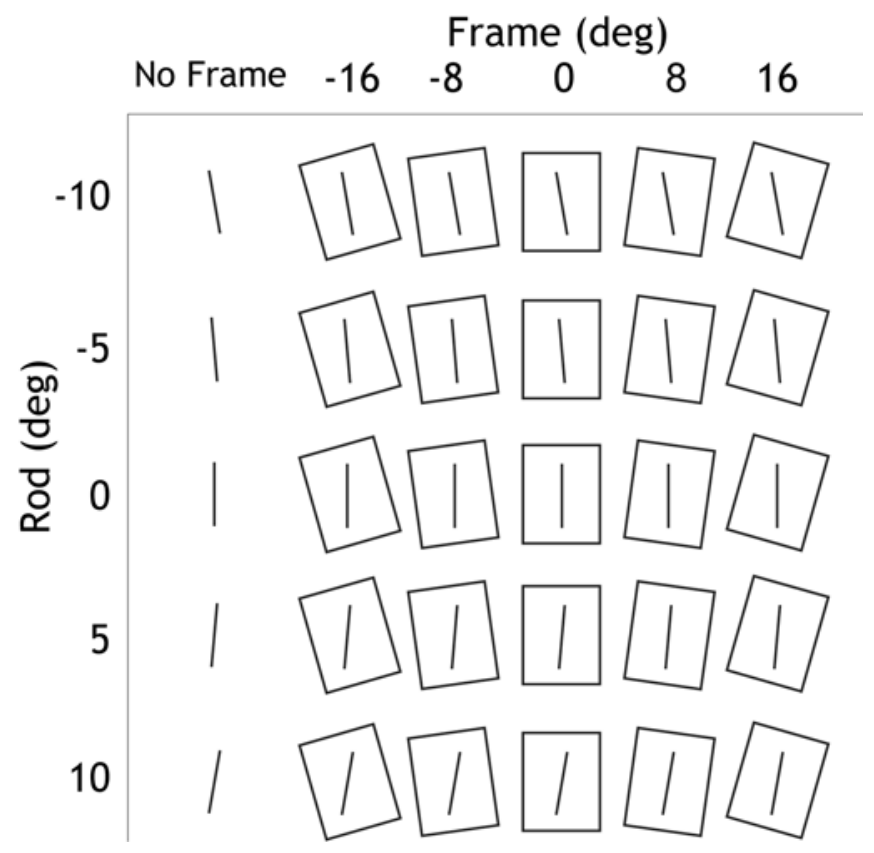

Figure 1 - Stimuli. Rows show the stimulus combinations with different rod orientations and columns show the stimulus combinations with different frame conditions. 
matching task. The study nicely demonstrates that different versions of orientation illusions can differentially affect visuomotor processing.

Dyde and Milner (2002) interpreted their findings in terms of the dorsal-ventral stream dichotomy as proposed by Milner and Goodale (1995). However, in all of their tasks, Dyde and Milner asked participants to produce a movement, the orientation of which had to be matched with an earlier perceived orientation of a part of the visual scene. Indeed, in most studies referred to earlier, contextual effects on the orientation perception were only measured in the last phase or at the end of the response. In studies that used grasping responses, usually the maximum aperture was analyzed, which occurs at about $70 \%$ of movement time (MT; Jeannerod, 1984). Yet, little is known about how these effects disperse over the entire response.

The task we used to scrutinize the evolution of the RFI is comparable to the aforementioned mailing task used by Dyde and Milner (2002). Participants were requested to rotate a hand-held cylinder into the same orientation as the orientation of the rod in the RFI. Rod and frame orientations were varied independently (see Figure 1), and the cylinder rotation was recorded by means of a 3-D motiontracking system.

Because it has been suggested that the presence or absence of visual feedback (De Grave et al., 2004; Glover \& Dixon, 2004) and stimulus-onset delays (Bridgeman et al., 1997; Westwood \& Goodale, 2003) can affect performance in perception-action responses to size-contrast or position illusions, these were also manipulated in the current experiment.

Effects of stimulus-response delays originate from the different latencies of the brain areas involved. The dorsal visual stream is known to have a short latency and stores a short-lived representation of veridical spatial stimulus properties. The ventral visual stream is involved in higher-order cognitive processes and has a longer latency, and its representations are more sensitive to context information that last longer (Rossetti, Pisella, \& Pelisson, 2000). This way, after a long delay, responses can only be guided by a ventral representation of the visual stimulus.

In our study we manipulated orientation information in a compound stimulus instead of size or position information, and because the orientation cues that we provided could be processed dorsally and ventrally, we did not have any clear prediction of the possible delay effects. Nevertheless, we thought it wise to include the parameter in our study to check whether differential effects as a function of the delay parameter would occur.

We analyzed the time-normalized orientation of the cylinder in the sagittal plane, and by applying multiple linear regression analyses to the data of each time sample, we were able to scrutinize the RFI effects in time. The beta weights for the regressor variables reflect the contribution of rod, frame, and starting orientations to the evolving cylinder orientation $(R y)$ during the responses.

At $t=0$ we expected no effects of rod and frame orientation because at that moment, the cylinder orientation was completely determined by the prescribed starting orientation Ryi $\left(b_{\text {Rod }}=0, b_{\text {Frame }}=0, b_{\text {Ryi }}=1\right)$. In contrast, at movement completion (i.e., at $t=100 \%$ ), the rod and frame effects were expected to be maximal, whereas the effect of $b_{\text {Ryi }}$ was expected to have worn off. The interesting part of the regression analyses covers the phase during the response, and especially the time course of the frame effects, because the frame orientation is a contextual variable that is irrelevant to the task (i.e., reproducing the perceived rod orienta- 
tion). Within the response period, effects were expected to kick in, increase, reach their maximum, and stabilize, with all such effects potentially being reflected in the respective beta weights of the regressor variables.

This approach enabled us to test whether the RFI stimulus was solely processed as a Gestalt or whether the individual components (rod and frame) that make up the Gestalt would play separate roles. There would be strong supporting evidence for the first option if rod and frame effects would occur simultaneously (i.e., rod and frame effects would kick in at the same time, have the same effect interval, and stabilize at the same time). However, if the processing of the RFI stimulus would somehow consist of multiple information processes dealing with the orientation of the rod and surrounding frame separately, then this should be revealed by target (rod) and context (frame) effects having different event structures.

\section{Method}

\section{Participants}

Fifteen right-handed subjects, seven men, volunteered to participate in our experiment for remuneration or course credit. All subjects had normal or corrected-tonormal vision and were unaware of the purpose of the experiment. Data of two participants were excluded because of technical problems and not complying to the task instructions. Age of the 13 participants who were included in the analyses ranged between 19 and 29 years (mean $=24, S D=3.6$ years). The local ethics committee approved the experimental protocol for the current experiment, and all subjects gave their written informed consent, as required by the 1964 Declaration of Helsinki.

\section{Material and Design}

Participants sat comfortably in a normal chair with arm rests facing a computer screen at a distance of approximately $1.5 \mathrm{~m}$. They were not restrained but were instructed to keep their back against the backrest of the chair during the experiment. To minimize external orientation cues, we covered the edges of the computer screen with a $100-\mathrm{cm}$ diameter black screen with a $28-\mathrm{cm}$ diameter circular opening in the center. In addition, the table on which the monitor stood was covered with black fabric, and during the experiment, the room was fully darkened.

Responses were given by moving a hand-held cylinder $($ diameter $=4 \mathrm{~cm}$, length $=20 \mathrm{~cm}$, weight $=222 \mathrm{~g}$ ). This cylinder was designed such that it could radiate white light as soon as the go signal sounded. To prevent reflection images on the stimulus presentation monitor, the side of the cylinder that faced toward the screen was covered with black sticky foil, leaving a $20 \mathrm{~cm} \times 2.2 \mathrm{~cm}$ area open and visible to the participant. The orientation and displacement of this cylinder were recorded by means of an Optotrak 3020 (Northern Digital, Waterloo, Canada) 3-D motion-tracking system using three infrared emitting diodes (IREDs) with a sampling frequency of $100 \mathrm{~Hz}$.

The stimuli consisted of a rod (height $\times$ width: $111 \times 8 \mathrm{~mm}, 4.2^{\circ}$ visual angle) in the center of the computer screen that was surrounded by a rectangular 
frame (height $\times$ width: $167 \times 131 \mathrm{~mm}, 6.4^{\circ} \times 2.5^{\circ}$ visual angle). Both rod and frame were visually presented in light gray on a black background. The center of the frame corresponded with the center of the rod. In contrast to mathematical conventions, clockwise $(\mathrm{cw})$ rotations were defined as positive rotations to increase the legibility of the results patterns.

There were five rod conditions $\left(-10^{\circ},-5^{\circ}, 0^{\circ}, 5^{\circ}\right.$, and $10^{\circ}$, relative to the gravitational vertical that was labeled $0^{\circ}$ ) and six frame conditions: in 1/6 of the trials the frame was absent, and in the remaining trials the rod was surrounded by a frame oriented in one of five possible orientations $\left(-16^{\circ},-8^{\circ}, 0^{\circ}, 8^{\circ}\right.$, and $\left.16^{\circ}\right)$. An overview of all possible stimuli is shown in Figure 1.

The interval between the mask that was shown after stimulus presentation and the go signal varied and was $17 \mathrm{~ms}$ for half the trials and $1000 \mathrm{~ms}$ for the other half of the trials. Start position (wrist pronated or supinated with the cylinder approximately horizontal) and visual feedback (i.e., cylinder light on or off) were varied between blocks and counterbalanced between participants. Every trial was repeated twice, and the experiment thus consisted of four blocks of 5 (Rod Orientations $) \times 6$ (Frame Conditions $) \times 2$ (Delay Conditions $) \times 2($ Replications $)=120$ trials each, in a quasi-randomized design.

\section{Procedure}

Participants received written and verbal instructions and performed a few practice trials before the experiment started. The aims of the practice trials were twofold: (1) familiarizing participants with the task and (2) testing and making sure that the cylinder trajectories were recorded by the Optotrak system.

Participants started each trial with the cylinder in their right hand and their forearm resting on the armrest, in either a pronated or supinated starting position. They were asked to keep the cylinder in a horizontal position. Brief verbal instructions about starting position and information about the visual feedback condition of every block were given before each block.

The trial events are shown in Figure 2. Stimuli were shown for $300 \mathrm{~ms}$, immediately followed by a semirandom masking pattern that was shown for $50 \mathrm{~ms}$ to prevent afterimages. The mask was 1 of 10 randomly chosen displays consisting of 50 randomly oriented and dispersed rods. The presentation of the mask was followed by a delay of either 17 or $1000 \mathrm{~ms}$ and completed with an auditory go signal. On this cue, participants were requested to make a smooth rotation and propelling movement with their right arm toward the screen such that, after stretching their arm in front of them, the orientation of the hand-held cylinder would occlude and accurately match the orientation of the stimulus rod that they had seen before. Participants had to keep the cylinder in this orientation until $2 \mathrm{~s}$ after the go signal a second tone indicated the end of the trial. After this, participants returned to their start position and waited for the next trial. Figure 2 shows the outline of the different trial events.

The whole experiment consisted of 480 trials with short breaks in between blocks. When necessary, participants were also allowed to take brief pauses in between trials. The experiment took about $1 \mathrm{hr}$ for each participant. 


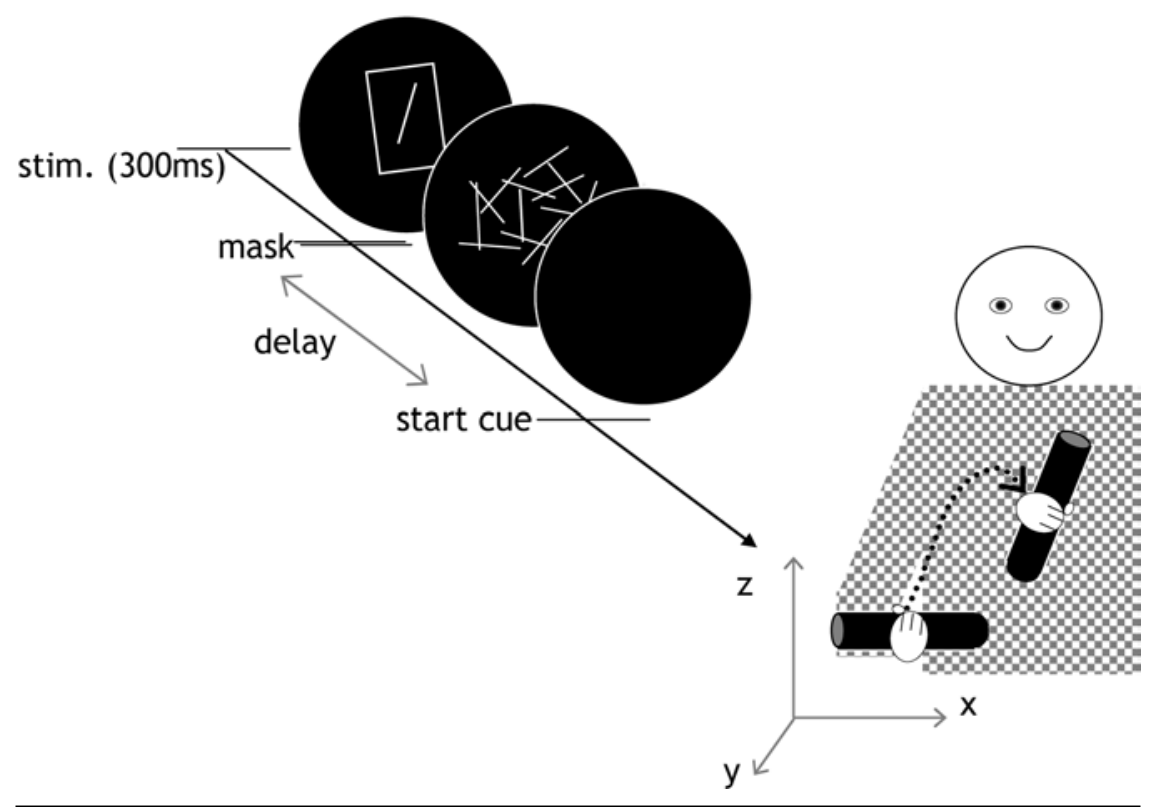

Figure 2 - Temporal outline of trial events.

\section{Data Analyses}

The three IREDs that were fixated onto the cylinder constructed a "Rigid Body" (Bouwhuisen, Meulenbroek, \& Thomassen, 2002) of which the $x, y$, and $z$ coordinates and the rotation around the sagittal axis $(R y)$ were analyzed. Data were filtered using a dual-pass, second-order, low-pass Butterworth filter with a cutoff frequency of $12 \mathrm{~Hz}$. Missing data were linearly interpolated. The beginning of the responses was defined as the first local minimum in the cylinder tangential velocity pattern that occurred before the moment at which the velocity increased beyond a threshold value of $5 \%$ of the maximal velocity in that trial. The end of the responses was defined as the first minimum in the cylinder tangential velocity pattern after the velocity had decreased below the threshold value of $5 \%$ of the maximal velocity in that trial. From these data, reaction time $(R T)$ and movement time (MT) were computed. $R T$ was defined as the duration of the interval between the go signal and response onset. $M T$ was defined as the duration of the interval between movement onset and the end of the response.

Signed error was calculated as the difference between the observed cylinder orientation at movement completion and the requested rod orientation, and this measure was taken to reflect the orientation bias induced by the rod and frame orientations of every stimulus. The effects of rod and frame orientations on signed error were analyzed by means of a Rod Orientation (5) $\times$ Frame Orientation (5) $\times$ Start Orientation (2) repeated-measures ANOVA, and the main effects of visual 
feedback, delay, and start condition on signed error were analyzed by means of paired-samples $t$ tests.

To enable comparisons of the $R y$ trajectories between trials and conditions, we time normalized $R y$ to 50 samples. To tease apart the rod and frame effects over time, the time-normalized movement trajectories were analyzed by means of linear regression analyses for every time sample $(t)$ using the model described in Equation 1.

$R y(t)=b_{0}(t)+b_{\mathrm{Ryi}}(t) \times R y i+b_{\mathrm{Rod}}(t) \times \operatorname{Rod}+b_{\text {Frame }}(t) \times$ Frame

$R y(t)$ represents the cylinder orientation at time sample $(t)$ and Ryi, Rod, and Frame the constant factors during every trial (i.e., the start orientation, the rod orientation, and the frame orientation, respectively). The beta weight $b_{\mathrm{Ryi}}(t)$ indicates how much the starting orientation contributes to the cylinder orientation at time sample $(t)$, and $b_{\text {Rod }}(t)$ and $b_{\text {Frame }}(t)$ are measures of the size of the contributions of rod and frame orientation to $R y$. In line with the task instructions, we expected $b_{\text {Ryi }}$ to be maximal at the beginning of the response and $b_{\text {Rod }}$ to be maximal at the end of the response.

\section{Results}

An example of the cylinder kinematics observed in one trial (supinated starting position, rod $=10^{\circ}$ and frame $=0^{\circ}$ ) is shown in Figure 3. The black solid line shows that the cylinder orientation in the frontoparallel plane $(R y)$ changes sigmoidally from $82^{\circ}$ to $-32^{\circ}$, showing an extreme overshoot of $22^{\circ}$. The rotations in

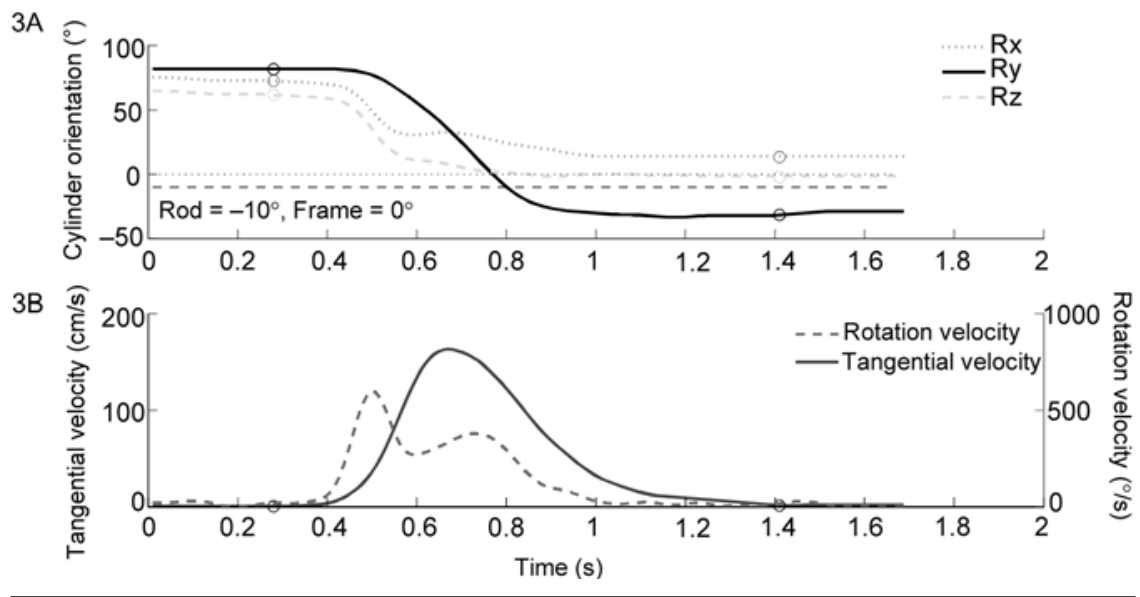

Figure 3 - Kinematics of the cylinder translations and rotations for a single trial, starting with a supinated starting posture. The top panel (A) shows cylinder rotations in time around the $x, y$, and $z$ axes, the dashed horizontal line shows the stimulus-rod orientation, and the dotted horizontal line represents the vertical. The bottom panel (B) shows the tangential and absolute rotational velocities of the cylinder in time. The latter velocity was calculated as the square root of the sum of the squared first derivatives of the three angular-position time functions shown in 3A. Circles indicate beginning and end of the movement. 
the sagittal $(R x)$ and transverse $(R z)$ planes were smaller and less smooth. The final orientations around the $x$ and $z$ axes were $13^{\circ}$ and $-2^{\circ}$, respectively.

Overall kinematic characteristics are listed in Table 1 . Mean $R T$ was $353 \mathrm{~ms}$ $(S D=68.2 \mathrm{~ms})$, and mean $M T$ was $1210 \mathrm{~ms}(S D=156.4 \mathrm{~ms})$. Given that grasping movements, on average, take between 650 and 900 ms (Marteniuk, Leavitt, Mackenzie, \& Athenes, 1990; Zaal \& Bootsma, 1993), the currently studied arm rotations can be said to be relatively slow. The average starting orientation $(R y i)$ was $-64.4^{\circ}\left(S D=14.7^{\circ}\right)$ in the pronated start condition and $72.6^{\circ}\left(S D=9.8^{\circ}\right)$ in the supinated start condition. Mean rotation amplitude $(R y$ [end] $-R y i)$ was $69.1^{\circ}(S D$ $\left.=16.4^{\circ}\right)$, and the average rotation velocity was $58.5^{\circ} / \mathrm{s}\left(S D=15.8^{\circ} / \mathrm{s}\right)$.

\section{Effects on Signed Error}

The mean signed error was $1.03^{\circ}\left(S D=7.9^{\circ}\right)$. The effects of the rod and frame orientations on the signed error (in degrees) are shown in Figures 4 and 5, respectively. Both show a sigmoidal pattern, with a positive slope for the effects of the rod orientation and a negative slope for the effects of the frame orientation.

Signed errors per rod orientation ranged from $4.7^{\circ}\left(S D=7.5^{\circ}\right)$ when it was $10^{\circ}$ tilted in the clockwise direction to $3.3^{\circ}\left(S D=8.3^{\circ}\right)$ when the rod had an orientation of $-10^{\circ}$. Thus, participants rotated the cylinder in the correct direction, but on average, the slant of the stimulus rod was overestimated.

Figure 5 shows the effects of frame orientation on the signed error. The negative slope of the sigmoidal pattern indicates that signed error was largest with a frame tilt of $-16^{\circ}\left(2.9^{\circ}, S D=7.6^{\circ}\right)$ and smallest with a frame tilt of $16^{\circ}\left(0.63^{\circ}, S D\right.$ $=7.7^{\circ}$ ).

A repeated-measures ANOVA according to a 5 Rod $\times 5$ Frame $\times 2$ Start orientation design showed that the signed error was affected by rod orientation $[F(4,48)=17.103, p<.01]$ as well as frame orientation $[F(4,48)=27.329, p<$ $.01]$. The two factors showed an interaction $[F(16,192)=2.272, p<.01]$ reflecting that signed errors caused by the contextual frame increased as the target rod orientation increased. In addition, interactions were found between rod and start condition $[F(4,48)=4.579, p<.05]$ and between frame and start condition $[F(4$, $48)=2.692, p<.05]$. However, starting position did not have a main effect on

\section{Table 1 Kinematic Characteristics of the Cylinder Rotations ${ }^{a}$}

\begin{tabular}{lrrrr}
\hline & Min & Max & Mean & \multicolumn{1}{c}{ SD } \\
\hline Reaction time $(\mathrm{ms})$ & 204.3 & 437.6 & 353.0 & 68.2 \\
Movement time $(\mathrm{ms})$ & 929.5 & 1425.2 & 1210.0 & 156.4 \\
Rotation amplitude $\left({ }^{\circ}\right)$ & 56.7 & 80.3 & 69.1 & 16.4 \\
Ryi pronated start $\left({ }^{\circ}\right)$ & -89.5 & -17.2 & -64.4 & 14.7 \\
Ryi supinated start $\left({ }^{\circ}\right)$ & 10.8 & 89.5 & 72.6 & 9.8 \\
Rye pronated start $\left({ }^{\circ}\right)$ & -30.7 & 36.1 & 1.4 & 11.6 \\
Rye supinated start $\left({ }^{\circ}\right)$ & -39.8 & 40.7 & 0.8 & 14.3 \\
Average rotation & 43.0 & 68.5 & 58.5 & 15.8 \\
velocity $(\%$ s) & & & & \\
\hline
\end{tabular}

a The statistics were calculated across participants. 


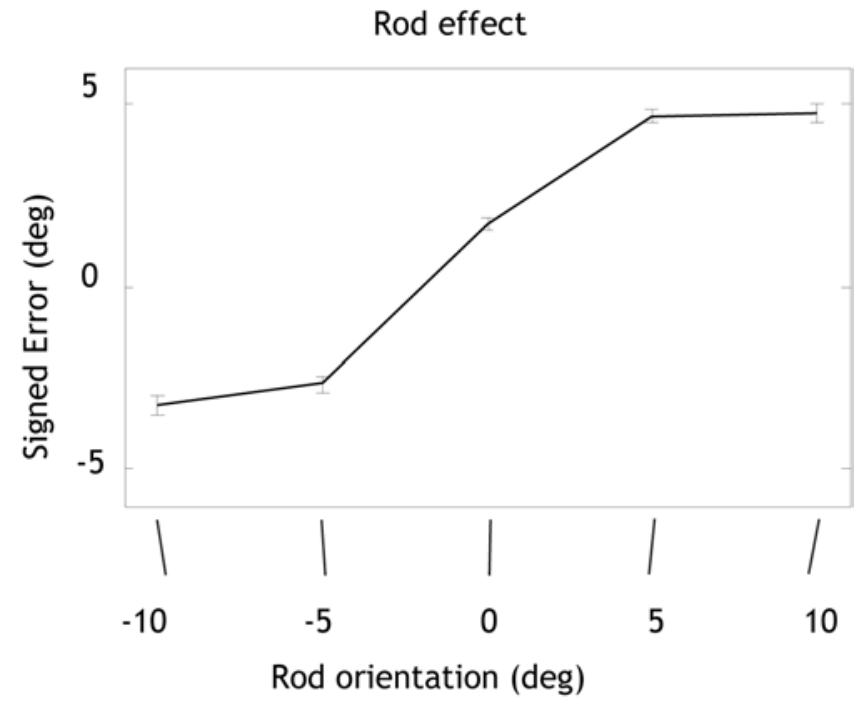

Figure 4 - Effects of rod orientation on the signed error averaged across participants and frame orientations. Error bars indicate standard errors.

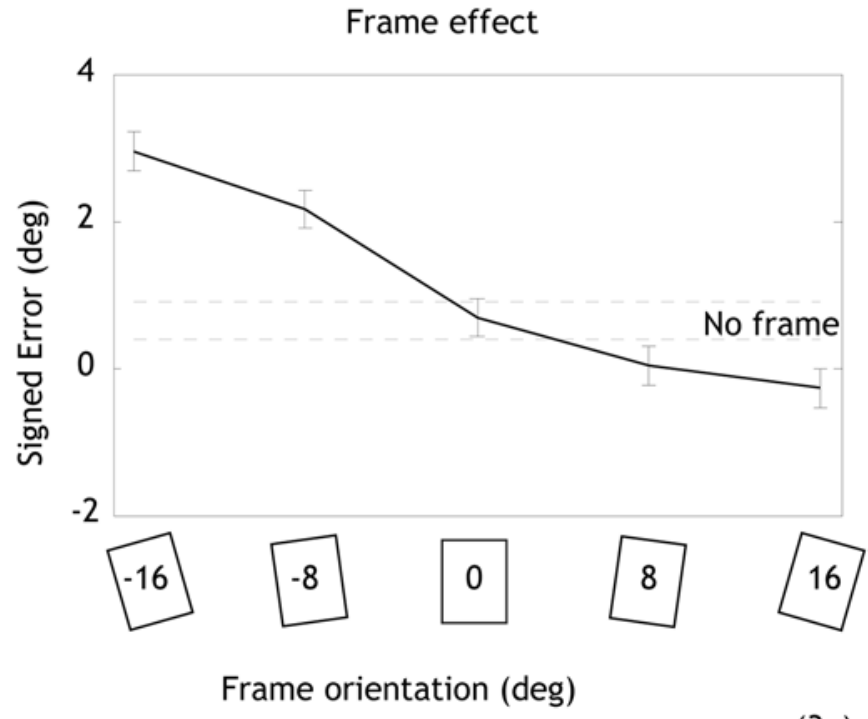

(3a)

Figure 5 - Effects of frame orientation on signed error averaged across participants and rod orientations. Error bars indicate standard errors, and the dashed lines the averaged signed error $\pm S E$ of the no-frame condition. 
signed error $[F(1,12)<1, \mathrm{~ns}]$. An additional paired-samples $t$ test showed no difference between the "no frame" and the frame $=0^{\circ}$ conditions $[t(12)<1$, ns]. Furthermore, signed error was not affected by visual feedback, delay, or start condition, as was shown by pairwise $t$ tests [all $t(12)<1$, ns]. For all further analyses, we pooled the data across visual feedback, delay, and start conditions.

\section{Rod and Frame Effects in Time}

To analyze the time course of the rod and frame effects during the responses, we analyzed the rotation of the cylinder in the frontoparallel plane $(R y)$. To be able to average across trials and to compute contrasts, $R y$ was normalized to time in 50 steps. Figure $3 \mathrm{~A}$ shows that $R y$ developed as a smooth sigmoidal pattern, in contrast to the cylinder rotations in the other directions. The tangential velocity as illustrated by Figure 3B shows a smooth bell-shaped pattern, whereas the rotation velocity has a multiphasic pattern with an early peak that is caused by noise in $R x$ and $R z$. We did not analyze $R x$ and $R z$ because the stimuli and the task instructions emphasized the frontoparallel plane as the plane in which the cylinder rotations needed to be realized.

To track the effects of the different experimental factors in time, we applied linear regression analyses for every normalized time sample. We used the linear regression model as described in Equation 1 and computed the beta weights $\left(b_{0}\right.$, $b_{\text {Ryi }}, b_{\text {Rod }}$, and $b_{\text {Frame }}$ ) for every time sample $(t)$ for every participant based on the data of all trials in which the frame was visible. A $b$ value of 0 means that this factor has no effect on $R y$, a positive $b$ value indicates a contribution to $R y$, and a negative $b$ value means that this factor has an effect on $R y$ in the opposite direction. In addition, the larger $b$ is, the stronger the contribution. The changes of the different $b$ values as a function of time are plotted in Figures 6B-D.

For a better understanding of the relation between the development of the different beta weights and the prehension response, we plotted the mean, timenormalized, tangential velocity in Figure 6A. Comparing the beta weights (Figure $6 \mathrm{~B}-\mathrm{D})$ with the tangential velocity (Figure 6A) shows that the rod and frame effects have stabilized before the response has finished completely.

$R^{2}$ per participant ranged from .58 to $.86($ mean $=.73, S D=.09)$. As expected, at $t=0 \%, R y$ was fully determined by $R y i$, whose contribution steadily decreased to 0 toward the end of the response (Figure $6 \mathrm{C}$ ). The effect of the rod orientation increased shortly after movement onset in a sigmoidal fashion (Figure 6A), whereas the negative effect of the frame-albeit smaller-increased in a sigmoidal fashion toward the end of the response (Figure 6B).

Because we were interested in the moments at which the effects started to kick in (T1) and reached their final levels (T2), we defined thresholds of 5\% of the range of $b_{\text {Frame }}$ and $b_{\text {Rod }}$ for every participant. (The 5\% threshold was arbitrary but both a lower [2.5\%] and a higher threshold [10\%] resulted in similar result patterns.) T1(rod) was considered to be the moment $b_{\text {Rod }} \geq$ threshold and T1(frame) to be the moment $b_{\text {Frame }} \leq-$ threshold. T2 values for each participant were defined as the moments at which the $b_{\text {Rod }} \geq b_{\text {Rod }}$ (end) - threshold and $b_{\text {Frame }} \leq b_{\text {Frame }}$ (end) + threshold.

T1 occurred on average at $29.8 \%(S D=8.3 \%)$ of $M T$ for rod and at $31.8 \%$ $(S D=16.6 \%)$ of $M T$ for frame $[t(12)<1, \mathrm{~ns}]$. T2 was later for rod effects $(79.1 \%$, 


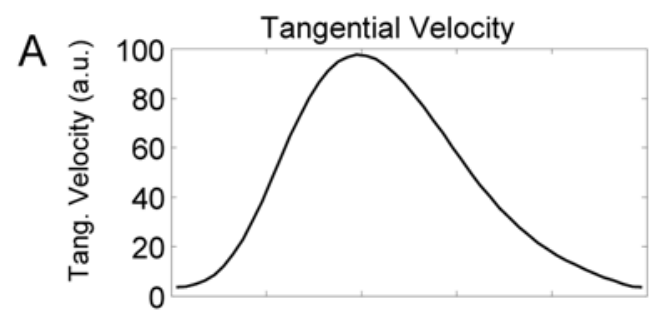

B
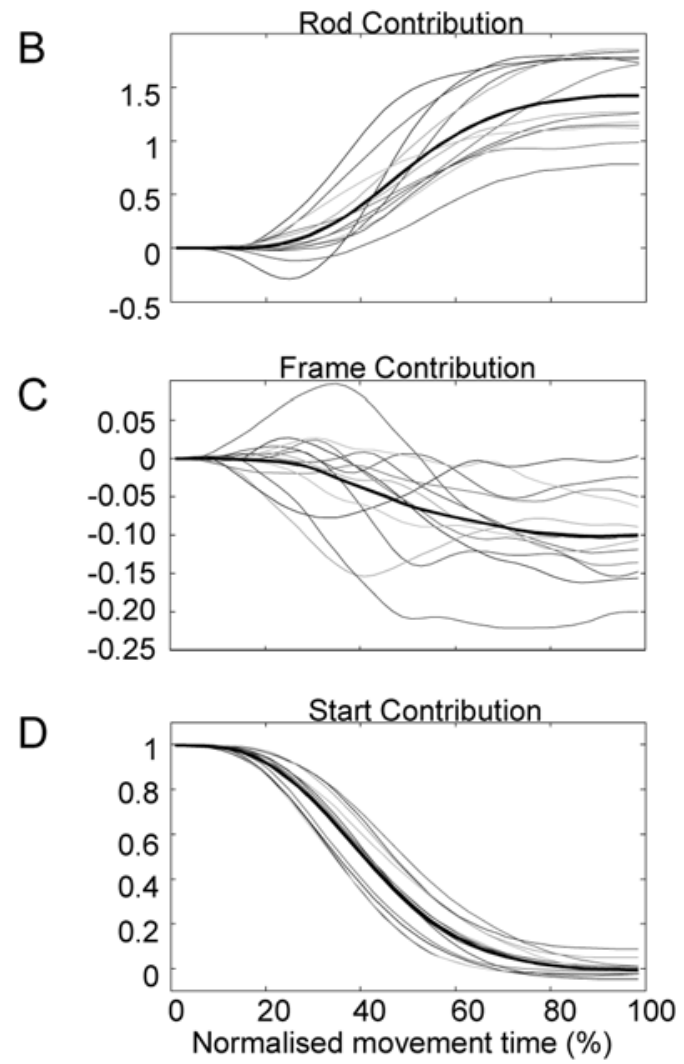

Figure 6 - The top panel shows (A) the mean time-normalized tangential velocity, and the lower three panels show the variations in beta weights in normalized time for the regression model: $R y=b_{0}+b_{\text {Ryi }} \times R y i+b_{\text {Rod }} \times \operatorname{Rod}+b_{\text {Frame }} \times$ Frame, reflecting the time courses of the contributions of the (B) rod, (C) frame, and (D) start orientations.

$S D=7.1 \%)$ as compared with frame effects $(64.3 \%, S D=26.0 \% ; t(12)=2.21, p$ $<.05)$. The effect interval (T2-T1) was longer for $\operatorname{rod}(49.2 \%, S D=9.3 \%)$ than for frame $(32.5 \%, S D=18.3 \% ; t(12)=3.26, p<.01)$.

By analyzing the moments at which the respective $b$ values became significant (Tsig), it showed that for 10 participants Tsig(Rod) $<$ Tsig(Frame), for 2 
participants $b_{\text {Frame }}$ became never significant, and 1 participant showed an opposite pattern $(\mathrm{n}+11, \mathrm{n}-2, p \leq .02)$.

In sum, analysis of the signed errors showed that both stimulus features caused errors: the rod orientations caused the final cylinder orientation to deviate in the same direction (i.e., the larger the rod deviation, the larger the effect in that direction), this in contrast to the frame, which contributed to the final cylinder orientation in the opposite direction. We found no main effects of visual feedback, delay, and starting position. The instruction to keep the cylinder horizontal in a supinated or pronated starting position was not completely followed. On average, participants held the cylinder a little less pronated or supinated, probably because those positions are more comfortable than the positions necessary to keep the cylinder in orientations of $-90^{\circ}$ and $+90^{\circ}$.

The interaction effects between starting position and rod orientation and between starting position and frame orientation can be summarized as follows: The rod and frame effect curves proved steeper for the supinated start condition than for the pronated start condition. Clearly, the extent to which the RFI affected the participants' forearm rotations depended on the initial starting posture that the participants were asked to adopt. This is likely the result of asymmetrical biomechanical properties of the right forearm (O'Sullivan \& Gallwey, 2005). The directional difference between rod and frame effects was also confirmed by the linear regression analyses performed on the RFI effects in time. These also showed that despite the fact that $b_{\text {Rod }}$ became significant earlier than $b_{\text {Frame, }}$ rod and frame effects kicked in (T1) simultaneously, whereas T2 occurred earlier for frame effects than for rod effects. As a consequence, the effect interval for rod was longer than for frame.

\section{Discussion}

We conducted the current study to gain a better understanding of the way in which forearm rotations toward a visually presented target orientation are biased by a tilted contextual frame. To do so we analyzed both the effects of the RFI on the orientation of a hand-held cylinder at the end of the requested forearm rotation and the development of the rod and frame effects during the forearm rotation.

In our study we demonstrated robust RFI effects on targeted forearm rotations, whereas the RFI study by Dyde and Milner (2002) suggested that a semantic context was a prerequisite for such effects to occur. The main difference from their study is the response that we requested from the participants. Dyde and Milner (2002) used grasping responses, and because the hand and fingers have many degrees of freedom, recording hand orientation might not be the best way to measure effects of an orientation illusion on grasping. More important, for grasping, other features might be more important than the orientation of the target. For example, Smeets and Brenner (1999) suggested that the locations where the fingers are to be placed on a to-be-grasped object are important for grasping responses. Thus, illusion effects are highly task dependent. Rather than trying to capture perception-action interactions by analyzing a collective behavioral variable, we have shown that scrutinizing the component processes that make up a visual illusion forms a powerful tool to gain more insights into the differential effects that visual illusions might have on action. 
Depending on the depth of processing, a compound visual stimulus can be processed as separate parts or as a whole. Although effects of rod and frame orientation kicked in at the same moment, their duration differed. On the basis of our rationale formulated in the introduction, we infer that, given the current task, the RFI stimulus was not solely processed as a Gestalt. If that were the case, then rod and frame effects should not only have kicked in at the same time, but their effects should also have worn off at the same time. Apparently the stimulus components influenced the forearm rotations separately. The fact that the frame effects stabilized sooner than the rod effects also demonstrates that the frame orientation was not a relevant cue with respect to the task instruction but was instead a distractor.

The task relevance of a stimulus property is indeed important for the way in which a stimulus is used for action. De Grave, Franz, and Gegenfurtner (2006), for example, showed that the Muller-Lyer illusion only affects movements when the illusory length is relevant to the task. In the current experiment, the rod orientation was relevant to the task but the frame orientation was not. However, we did find a systematic effect of the frame orientation on the cylinder rotations. This suggests that orientation is more sensitive to contextual distractors than other spatial stimulus properties such as position and length information in a pointing task (De Grave et al., 2006).

The finding that the frame effects stabilized sooner than the rod effects suggests that in the homing-in phase, or at least toward the end of the responses, a more veridical representation of the stimulus orientation determined the adopted final orientation of the hand-held cylinder. Surprisingly, the rod effects also stabilized long before the end of the responses (about $250 \mathrm{~ms}$ ). The rotational velocity profiles show subtle fluctuations at the beginning and end of the prehension responses. Apparently some rotational adjustments did occur during the start and homing-in phases of the response. At the end of the movement, the rod orientation is most important; therefore, the adjustments that occur in that phase are possibly only guided by the represented rod orientation.

We explored possible explanations for the constant clockwise offset in the realized final cylinder orientations by comparing the signed error data in the presence and absence of the frame and, similarly, in the presence and absence of visual feedback from the cylinder, but the overall rotational bias proved to be independent of these experimental factors. We, therefore, assume the offset to be likely caused by anatomical or proprioceptive factors that bias the response execution. It should be noted that, to make a stronger statement about the nature (perceptual versus motoric) of these errors, a purely perceptual measure would be necessary. That is, however, beyond the scope of this study.

Our findings are in line with the Roelofs effect in which a laterally shifted frame induces a pointing bias in the direction opposite to that of the frame shift (Bridgeman et al., 1997). Interestingly, some participants in Bridgeman et al.'s study showed such biased pointing responses only after a long delay ( $>4 \mathrm{~s})$, whereas the responses of other participants were biased by the frame in all delay conditions. Bridgeman et al. (1997) took this as support for different stimulus representations being involved in the sensorimotor maps that the participants used to control their pointing movements. The fact that we did not find any effects of stimulus-response delay is either the result of the fact that position and orientation information are represented in different ways in the visuomotor system or of dif- 
ferences in the task requirements between the pointing movements studied in the context of the Roelofs effect and the currently studied forearm rotations in the context of the RFI. Clearly, an oriented rod is a more complex stimulus than a single dot at a certain planar position. To accurately perceive and remember the orientation of a visually presented rod, unambiguous contextual cues are helpful.

Another difference is that the forearm rotations studied here apparently are more complex than pointing responses, which might be prepared while the stimulus location is still represented in the "veridical" dorsal visual stream. The reproduction of an earlier perceived orientation, however, requires the integration of more information, which takes more time and is cognitively more demanding and, thus, is likely dependent on a visual representation from the ventral visual stream.

We conclude that: (1) the rod and frame effects of the RFI as investigated by means of the kinematics of targeted forearm rotations can be distinguished in time, (2) the rod and frame effects of the RFI have identical latencies but the rod has a longer effect interval, and (3) the effects that orientation illusions have on motor performance are task dependent.

\section{Acknowledgments}

We would like to thank two anonymous reviewers for their helpful comments and S. van Pelt for a good suggestion for the analyses.

\section{References}

Aglioti, S., Desouza, J.F.X., \& Goodale, M.A. (1995). Size-contrast illusions deceive the eye but not the hand. Current Biology, 5(6), 679-685.

Antonucci, G., Fanzon, D., Spinelli, D., \& Zoccolotti, P. (1995). Visual factors affecting the rod-and-frame illusion: Influence of gap size and frame components. Perception, $24,1119-1130$.

Beh, H.C., \& Wenderoth, P.M. (1972). The effect of variation of frame shape on the angular function of the rod-and-frame illusion. Perception \& Psychophysics, 11(1A), 35-37.

Beh, H.C., Wenderoth, P.M., \& Purcell, A.T. (1971). The angular function of a rod-andframe illusion. Perception \& Psychophysics, 9(4), 353-355.

Bouwhuisen, C.F., Meulenbroek, R.G.J., \& Thomassen, A.J.W.M. (2002). A 3D motiontracking method in graphonomic research: Possible applications in future handwriting recognition studies. Pattern Recognition, 35, 1039-1047.

Bridgeman, B., Peery, S., \& Anand, S. (1997). Interaction of cognitive and sensorimotor maps of visual space. Perception \& Psychophysics, 59(3), 456-469.

De Grave, D.D.J., Brenner, E., \& Smeets, J.B.J. (2004). Illusions as a tool to study the coding of pointing movements. Experimental Brain Research, 155(1), 56-62.

De Grave, D.D.J., Franz, V.H., \& Gegenfurtner, K.R. (2006). The influence of the Brentano illusion on eye and hand movements. Journal of Vision (Charlottesville, Va.), 6(7), 727-738.

Dyde, R.T., \& Milner, A.D. (2002). Two illusions of perceived orientation: One fools all of the people some of the time; the other fools all of the people all of the time. Experimental Brain Research, 144, 518-527.

Fischer, M.H. (2001). How sensitive is hand transport to illusory context effects? Experimental Brain Research, 136, 224-230. 
Franz, V.H., Fahle, M., Bülthoff, H.H., \& Gegenfurtner, K.R. (2001). Effects of visual illusions on grasping. Journal of Experimental Psychology. Human Perception and Performance, 27(5), 1124-1144.

Franz, V.H., Gegenfurtner, K.R., Bülthoff, H.H., \& Fahle, M. (2000). Grasping visual illusions-No evidence for a dissociation between perception and action. Psychological Science, 11(1), 20-25.

Glover, S., \& Dixon, P. (2004). A step and a hop on the Muller-Lyer: Illusion effects on lower-limb movements. Experimental Brain Research, 154(4), 504-512.

Heath, M., Rival, C., \& Binsted, G. (2004). Can the motor system resolve a premovement bias in grip aperture? Online analysis of grasping the Muller-Lyer illusion. Experimental Brain Research, 158, 378-384.

Jackson, S.R., \& Shaw, A. (2000). The Ponzo illusion affects grip force but not grip aperture scaling during prehension movements. Journal of Experimental Psychology. Human Perception and Performance, 26(1), 418-423.

Jeannerod, M. (1984). The timing of natural prehension movements. Journal of Motor Behavior, 16, 235-254.

Marteniuk, R.G., Leavitt, J.L., Mackenzie, C.L., \& Athenes, S. (1990). Functional-relationships between grasp and transport components in a prehension task. Human Movement Science, 9(2), 149-176.

Milner, A.D., \& Goodale, M.A. (1995). The visual brain in action. Oxford: Oxford University Press.

O'Sullivan, L.W., \& Gallwey, T.J. (2005). Forearm torque strengths and discomfort profiles in pronation and supination. Ergonomics, 48(6), 703-721.

Rossetti, Y., Pisella, L., \& Pelisson, D. (2000). New insights on eye blindness and hand sight: Temporal constraints of visuo-motor networks. Visual Cognition, 7(6), 785808.

Smeets, J.B.J., \& Brenner, E. (1999). A new view on grasping. Motor Control, 3, 237271.

van Donkelaar, P. (1999). Pointing movements are affected by size-contrast illusions. Experimental Brain Research, 125, 517-520.

Westwood, D.A., \& Goodale, M.A. (2003). Perceptual illusion and the real-time control of action. Spatial Vision, 16(3-4), 243-254.

Zaal, F., \& Bootsma, R.J. (1993). Accuracy demands in natural prehension. Human Movement Science, 12(3), 339-345. 